Pacific

Journal of

Mathematics

\title{
ON THE HALF-SPACE THEOREM FOR MINIMAL SURFACES IN HEISENBERG SPACE
}

Tristan Alex 


\title{
ON THE HALF-SPACE THEOREM FOR MINIMAL SURFACES IN HEISENBERG SPACE
}

\author{
TRISTAN ALEX
}

\begin{abstract}
We propose a simple proof of the vertical half-space theorem for Heisenberg space.
\end{abstract}

\section{Introduction}

A half-space theorem states that the only properly immersed minimal surface which is contained in a half-space is a parallel translate of the boundary of the half-space, namely a plane. Hoffman and Meeks [1990] first proved it for $\mathbb{R}^{3}$. It fails in $\mathbb{R}^{n}$ or $\mathbb{U}^{n}$ for $n \geq 4$.

In recent years, there has been increased interest in homogeneous 3-manifolds (see [Abresch and Rosenberg 2004; Hauswirth et al. 2008]). The original proof of Hoffman and Meeks also works in Heisenberg space $\mathrm{Nil}_{3}$ with respect to umbrellas, which are the exponential image of a horizontal tangent plane [Abresch and Rosenberg 2005]. Daniel and Hauswirth [2009] extended the theorem to vertical half-spaces of Heisenberg space, where vertical planes are defined as the inverse image of a straight line in the base of the Riemannian fibration $\mathrm{Nil}_{3} \rightarrow \mathbb{R}^{2}$.

Vertical half-space theorem in Heisenberg space [Daniel and Hauswirth 2009]. Let $S$ be a properly immersed minimal surface in Heisenberg space. If $S$ lies to one side of a vertical plane $P$, then $S$ is a plane parallel to $P$.

Essential for the proof of half-space theorems is the existence of a family of catenoids or generalized catenoids. Their existence is simple to establish in spaces where they can be represented as ODE solutions. For instance, horizontal umbrellas in Heisenberg space are invariant under rotations around the vertical axis, so they lead to an ODE. However, the lack of rotations about horizontal axes means that the existence of analogues of a horizontal catenoid amounts to establishing true PDE solutions. Daniel and Hauswirth use a Weierstraß-type representation to reduce this problem to a system of ODEs. Only after solving a period problem do they obtain the desired family of surfaces.

MSC2010: primary 53A10, 53C42; secondary 53A35.

Keywords: minimal immersions, half-space theorem, Heisenberg space. 
In the present paper we introduce a simpler approach: we take a coordinate model of Heisenberg space and consider coordinate surfaces of revolution. Provided we can choose a family of surfaces whose mean curvature normal points into the half-space, the original maximum principle argument of Hoffman and Meeks will prove the theorem. Our approach is based on an idea by Bergner [2010], who generalized the classical half-space theorem to surfaces with negative Gaussian curvature such that the principal curvatures satisfy an inequality, and Sá Earp and Toubiana [1995], who consider special Weingarten surfaces with mean curvature satisfying an inequality.

It is an open problem to prove a vertical half-space theorem for $\mathrm{PSL}_{2}(\mathbb{R})$, where it would apply to surfaces whose mean curvature is the so-called magic number $H_{0}=1 / 2$, namely the limiting value of the mean curvature of large spheres. Here, it would state that surfaces with mean curvature $H_{0}=1 / 2$ lying on the mean convex side of a horocylinder can only be horocylinders, that is, the inverse image of a horocycle of the fibration $\mathrm{PSL}_{2}(\mathbb{R}) \rightarrow \mathbb{H}^{2}$. Our strategy could also work there. However, so far we have not been successful in establishing the desired family of generalized catenoids with $H \leq H_{0}$.

\section{The Euclidean half-space theorem}

Euclidean half-space theorem [Hoffman and Meeks 1990]. A properly immersed minimal surface $S$ in $\mathbb{R}^{3}$ lying in a half-space $H$ is a plane parallel to $P=\partial H$.

Proof. By the standard maximum principle we can assume $\operatorname{dist}(S, P)=0$ but $S \cap P=\varnothing$.

Let $\mathscr{C}_{r} \subset \mathbb{R}^{3} \backslash H$ be a half catenoid with necksize $r$ and $\partial \mathscr{C}_{r} \subset P$. By the properness of $S$, we can translate $S$ by $\varepsilon>0$ towards $\mathscr{C}_{1}$ such that $S$ intersects $P$ but stays disjoint to $\partial \mathscr{C}_{r}$ for all $r \in(0,1]$.

As $r$ tends to 0 , the family of catenoids $\mathscr{C}_{r}$ converges to $P$ minus a point. We claim that the set $I$ of parameters for which $\mathscr{C}_{r}$ does not intersect $S$ is open. Consider a catenoid $\mathscr{C}_{r_{0}}$ that does not intersect $S$. For each $r \in(0,1)$ there exists a compact set $K$ such that the distance between $\mathscr{C}_{r}$ and $P$ is larger than $2 \varepsilon$ in the complement of $K$. We may choose $K$ in a way that this property holds for all $r$ in a small neighborhood of $r_{0}$. This implies that the distance between $S$ and all these $\mathscr{C}_{r}$ is larger than $\varepsilon$ in the complement of $K$ (see Figure 1).

However, within the compact set $K$, the distance between $S$ and $C_{r_{0}}$ is positive, so for all $r$ in a (possibly smaller) neighborhood of $r_{0}$, this distance is still positive.

We conclude that in a small neighborhood of $r_{0}$,

$$
\operatorname{dist}\left(\mathscr{C}_{r}, S\right) \geq \min \left\{\operatorname{dist}\left(\mathscr{C}_{r} \cap K, S \cap K\right), \operatorname{dist}\left(\mathscr{C}_{r} \cap K^{c}, S \cap K^{c}\right)\right\}>0,
$$

thereby proving our claim. 


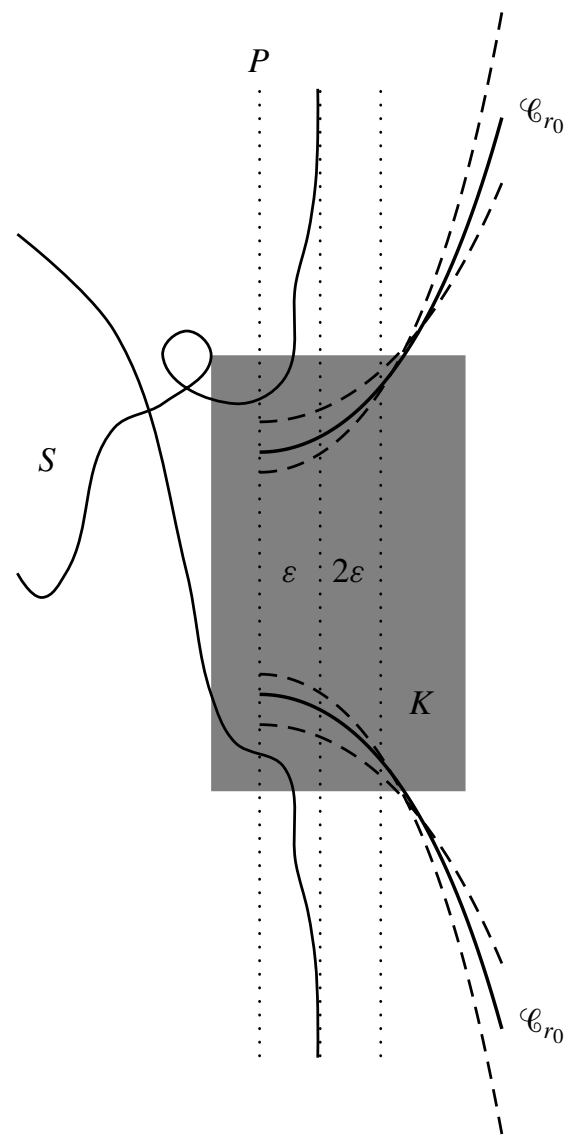

Figure 1. Proof of the Euclidean half-space theorem.

Therefore, the set of parameters for which $\mathscr{b}_{r}$ and $S$ do intersect is closed, so there is a first catenoid $\mathscr{C}_{r_{1}}$ touching $S$ at a point $p$. Since the boundaries of all $\mathscr{C}_{r}$ with $r \in(0,1]$ are disjoint from $S$, the touching point $p$ is an interior point, contradicting the maximum principle.

\section{Coordinate surfaces of revolution}

We take the following coordinates:

$$
\mathrm{Nil}_{3}:=\left(\mathbb{R}^{3}, \mathrm{~d} s^{2}\right), \quad \mathrm{d} s^{2}=\mathrm{d} x^{2}+\mathrm{d} y^{2}+(2 \tau x \mathrm{~d} y-\mathrm{d} z)^{2} \quad \text { with } \geq 0 .
$$

An orthonormal frame of the tangent space is given by

$$
E_{1}=\partial_{x}, \quad E_{2}=\partial_{y}+2 \tau x \partial_{z}, \quad E_{3}=\partial_{z},
$$


and the Riemannian connection in these coordinates is determined by

$$
\begin{array}{ll}
\nabla_{E_{1}} E_{2}=-\nabla_{E_{2}} E_{1}=\tau E_{3}, & \nabla_{E_{1}} E_{3}=\nabla_{E_{3}} E_{1}=-\tau E_{2}, \\
\nabla_{E_{2}} E_{3}=\nabla_{E_{3}} E_{2}=\tau E_{1}, & \nabla_{E_{i}} E_{j}=0 \text { in all other cases. }
\end{array}
$$

The Heisenberg space is a Riemannian fibration $\pi: \mathbb{R}^{3} \rightarrow \mathbb{R}^{2}$ with vanishing base curvature. The bundle curvature of $\mathrm{Nil}_{3}$ is given by $\frac{1}{2} g\left(\nabla_{E_{1}} E_{2}-\nabla_{E_{2}} E_{1}, E_{3}\right)=\tau$ and for $\tau=0$ we recover $\mathbb{R}^{3}$.

Let us consider a curve $c(t)=(0, t, r(t))$ in Heisenberg space with a positive function $r$ and $t \geq 0$. By rotating around the $y$-axis, we get an immersion

$$
f:\left[t_{0}, \infty\right) \times[0,2 \pi) \rightarrow \mathrm{Nil}_{3}, \quad(t, \varphi) \mapsto\left(\begin{array}{c}
-r(t) \sin \varphi \\
t \\
r(t) \cos \varphi
\end{array}\right) .
$$

In order to apply the proof of Hoffman and Meeks, we will construct Euclidean surfaces of revolution around the $y$-axis. With the Heisenberg space metric, these rotations are not isometric, because the 4-dimensional isometry group of $\mathrm{Nil}_{3}$ contains only translations and rotations around the vertical axis. Therefore, the mean curvature of such a surface will depend on the angle of rotation $\varphi$. We will need to find a surface with mean curvature vector pointing to the half-space to arrive at the desired contradiction with the maximum principle.

The tangent space of $M:=f\left(\left[t_{0}, \infty\right) \times[0,2 \pi)\right)$ is spanned by

$$
\begin{aligned}
& v_{1}=-r^{\prime}(t) \sin \varphi E_{1}+E_{2}+\left(2 \tau r(t) \sin \varphi+r^{\prime}(t) \cos \varphi\right) E_{3}, \\
& v_{2}=-r(t) \cos \varphi E_{1}-r(t) \sin \varphi E_{3},
\end{aligned}
$$

so the inner normal of $M$ is

$$
N=\frac{1}{W}\left(\sin \varphi E_{1}+\left(r^{\prime}(t)+2 \tau r(t) \sin \varphi \cos \varphi\right) E_{2}-\cos \varphi E_{3}\right),
$$

where $W=\sqrt{1+\left(2 \tau r(t) \sin \varphi \cos \varphi+r^{\prime}(t)\right)^{2}}$.

We will now compute the first and second fundamental forms of $M$. We easily get

$$
\begin{aligned}
G_{i j} & =\mathrm{d} s^{2}\left(v_{i}, v_{j}\right) \\
& =\left(\begin{array}{cc}
r^{\prime}(t)^{2} \sin ^{2} \varphi+\left(2 \tau r(t) \sin \varphi+r^{\prime}(t) \cos \varphi\right)^{2}+1 & -2 \tau r(t)^{2} \sin ^{2} \varphi \\
-2 \tau r(t)^{2} \sin ^{2} \varphi & r(t)^{2}
\end{array}\right)
\end{aligned}
$$

with determinant $\operatorname{det} G=r(t)^{2} W^{2}$.

The most tedious part of the calculation is the second fundamental form. We have to compute

$$
B_{i j}=\mathrm{d} s^{2}\left(\nabla_{v_{i}} v_{j}, N\right)
$$


To start, (1) gives

$$
\begin{aligned}
& \nabla_{v_{1}} E_{1}=\left(-2 \tau^{2} r(t) \sin \varphi-\tau r^{\prime}(t) \cos \varphi\right) E_{2}-\tau E_{3}, \\
& \nabla_{v_{1}} E_{2}=\left(2 \tau^{2} r(t) \sin \varphi+\tau r^{\prime}(t) \cos \varphi\right) E_{1}-\tau r^{\prime}(t) \sin \varphi E_{3}, \\
& \nabla_{v_{1}} E_{3}=\tau E_{1}+\tau r^{\prime}(t) \sin \varphi E_{2} .
\end{aligned}
$$

We calculate

$$
\begin{aligned}
\nabla_{v_{1}} v_{1}=- & r^{\prime \prime}(t) \sin \varphi E_{1}+\left(2 \tau r^{\prime}(t) \sin \varphi+r^{\prime \prime}(t) \cos \varphi\right) E_{3} \\
& -r^{\prime}(t) \sin \varphi \nabla_{v_{1}} E_{1}+\nabla_{v_{1}} E_{2}+\left(2 \tau r(t) \sin \varphi+r^{\prime}(t) \cos \varphi\right) \nabla_{v_{1}} E_{3} \\
=( & \left.r^{\prime \prime}(t) \sin \varphi+4 \tau^{2} r(t) \sin \varphi+2 \tau r^{\prime}(t) \cos \varphi\right) E_{1} \\
& +\left(4 \tau^{2} r(t) r^{\prime}(t) \sin ^{2} \varphi+2 \tau r^{\prime}(t)^{2} \sin \varphi \cos \varphi\right) E_{2} \\
& +\left(2 \tau r^{\prime}(t) \sin \varphi+r^{\prime \prime}(t) \cos \varphi\right) E_{3},
\end{aligned}
$$

and obtain the first entry of $B$ as

$$
\begin{aligned}
B_{11}=\frac{1}{W}(- & r^{\prime \prime}(t)+4 \tau^{2} r(t) r^{\prime}(t)^{2} \sin ^{2} \varphi+8 \tau^{3} r(t)^{2} r^{\prime}(t) \sin ^{3} \varphi \cos \varphi \\
& \left.+4 \tau^{2} r(t) \sin ^{2} \varphi+2 \tau r^{\prime}(t)^{3} \sin \varphi \cos \varphi+4 \tau^{2} r(t) r^{\prime}(t)^{2} \sin ^{2} \varphi \cos ^{2} \varphi\right) .
\end{aligned}
$$

The other three entries arise similarly from

$$
\begin{aligned}
& \nabla_{v_{2}} v_{1}=\nabla_{v_{1}} v_{2}=-\left(\tau r(t) \sin \varphi+r^{\prime}(t) \cos \varphi\right) E_{1} \\
&+\left(\tau r(t)\left(2 \tau r(t) \sin \varphi \cos \varphi+r^{\prime}(t) \cos (2 \varphi)\right)\right) E_{2} \\
&+\left(\tau r(t) \cos \varphi-r^{\prime}(t) \sin \varphi\right) E_{3}, \\
& \nabla_{v_{2}} v_{2}=r(t) \sin \varphi E_{1}-2 \tau r(t)^{2} \sin \varphi \cos \varphi E_{2}-r(t) \cos \varphi E_{3} .
\end{aligned}
$$

They are

$$
\begin{aligned}
B_{12}=B_{21}= & \frac{1}{W}\left(\tau r ( t ) \left(4 \tau r(t) r^{\prime}(t) \sin \varphi \cos ^{3} \varphi\right.\right. \\
& \left.\left.+\tau^{2} r(t)^{2} \sin ^{2}(2 \varphi)+r^{\prime}(t)^{2} \cos (2 \varphi)-1\right)\right), \\
B_{22}=-\frac{1}{W}( & \left.r(t)\left(\tau r(t) \sin (2 \varphi)\left(\tau r(t) \sin (2 \varphi)+r^{\prime}(t)\right)-1\right)\right) .
\end{aligned}
$$

We obtain the mean curvature $H$ for our coordinate surface of revolution:

Lemma 1. The mean curvature $H=H(t, \varphi)$ of $f$ is given by

$$
\begin{aligned}
H & :=\frac{1}{2} \operatorname{tr}\left(G^{-1} B\right) \\
& =\frac{G_{22} B_{11}-G_{12} B_{21}-G_{21} B_{12}+G_{11} B_{22}}{2 r(t)^{2} W^{2}} \\
& =\frac{1+r^{\prime}(t)^{2}-r(t) r^{\prime \prime}(t)+4 \tau^{2} r(t)^{2} \sin ^{4} \varphi+2 \tau r(t) r^{\prime}(t) \sin \varphi \cos \varphi}{2 r(t) W^{3}} .
\end{aligned}
$$




\section{Half-space theorem in Heisenberg space}

As expected, for $\tau=0$, Lemma 1 recovers the mean curvature for surfaces of revolution in Euclidean space. For $\tau \neq 0$, the two additional terms depending on $\varphi$ in the nominator of $H$ arise because the horizontal rotation is not an isometry of Heisenberg space. Our goal is to exhibit a family of surfaces of revolution satisfying $H \leq 0$ with respect to the normal $N$.

Consider the surface of revolution $f_{c}$ generated by the curve by

$$
r_{c}(t):=\exp \left(\frac{1}{c} \exp (c t)\right)
$$

with $c>c_{0}:=4 \tau^{2}+2 \tau+1$. We claim that this surface satisfies $H \leq 0$ for $t>0$. Indeed, the following estimate for the denominator of $H$ holds:

$$
\begin{aligned}
2 r(t) W^{3} H & \leq 1+r_{c}^{\prime}(t)^{2}-r_{c}(t) r_{c}^{\prime \prime}(t)+4 \tau^{2} r_{c}(t)^{2}+2 \tau r_{c}(t) r_{c}^{\prime}(t) \\
& =1+r_{c}(t)^{2}\left(\exp (c t)(2 \tau-c)+4 \tau^{2}\right) \\
& \leq 1+r_{c}(t)^{2}\left(4 \tau^{2}+2 \tau-c\right) \leq 1+4 \tau^{2}+2 \tau-c \leq 0 .
\end{aligned}
$$

Since we consider a surface of revolution with an embedded meridian, the embeddedness of $M_{c}:=f_{c}\left(\left[t_{0}, \infty\right) \times[0,2 \pi)\right)$ is obvious. Also, the boundary

$$
\partial M_{c}=\left\{\exp \left(\frac{1}{c}\right) \cdot(\sin \varphi, 0, \cos \varphi): \varphi \in[0,2 \pi)\right\}
$$

is explicitly known.

It is also important to note that for each $c$ and any given $\varepsilon>0$, there exists a compact set such that the distance between $M_{c}$ and the plane $\{y=0\}$ is larger than $\varepsilon$ in the complement of this compact set.

Let us summarize the result:

Lemma 2. The coordinate surface of revolution whose meridian is defined by (2) satisfies, for $c>c_{0}$,

(1) $H \leq 0$ with respect to the normal $N$,

(2) for $c \rightarrow \infty$, the surface $M_{c}$ converges uniformly to a subset of $\{y=0\}$ on compact sets,

(3) $M_{c}$ is properly embedded, and

(4) $\partial M_{c}=\left\{\exp \left(\frac{1}{c}\right) \cdot(\sin \varphi, 0, \cos \varphi): \varphi \in[0,2 \pi)\right\}$ for all $c$.

Using the surfaces $M_{c}$, our proof of the Euclidean half-space theorem literally applies to Heisenberg space.

\section{Acknowledgement}

I would like to thank my advisor Karsten Große-Brauckmann for his help. 


\section{References}

[Abresch and Rosenberg 2004] U. Abresch and H. Rosenberg, "A Hopf differential for constant mean curvature surfaces in $\mathbb{S}^{2} \times \mathbb{R}$ and $\mathbb{H}^{2} \times \mathbb{R}^{\prime}$, Acta Math. 193:2 (2004), 141-174. MR 2006h:53003 Zbl 1078.53053

[Abresch and Rosenberg 2005] U. Abresch and H. Rosenberg, "Generalized Hopf differentials", Mat. Contemp. 28 (2005), 1-28. MR 2006h:53004 Zbl 1118.53036

[Bergner 2010] M. Bergner, "A halfspace theorem for proper, negatively curved immersions", Ann. Global Anal. Geom. 38:2 (2010), 191-199. MR 2011j:53009 Zbl 1217.53010

[Daniel and Hauswirth 2009] B. Daniel and L. Hauswirth, "Half-space theorem, embedded minimal annuli and minimal graphs in the Heisenberg group", Proc. Lond. Math. Soc. (3) 98:2 (2009), 445-470. MR 2009m:53018 Zbl 1163.53036

[Hauswirth et al. 2008] L. Hauswirth, H. Rosenberg, and J. Spruck, "On complete mean curvature 1/2 surfaces in $\mathbb{H}^{2} \times \mathbb{R} ”$, Comm. Anal. Geom. 16:5 (2008), 989-1005. MR 2010d:53009 Zbl 1166.53041

[Hoffman and Meeks 1990] D. Hoffman and W. H. Meeks, III, "The strong halfspace theorem for minimal surfaces”, Invent. Math. 101:2 (1990), 373-377. MR 92e:53010 Zbl 0722.53054

[Sá Earp and Toubiana 1995] R. Sá Earp and E. Toubiana, "Sur les surfaces de Weingarten spéciales de type minimal”, Bol. Soc. Brasil. Mat. (N.S.) 26:2 (1995), 129-148. MR 96j:53006 Zbl 0864.53004

Received September 16, 2015. Revised October 30, 2015.

TRISTAN ALEX

FACHBEREICH MATHEMATIK

TECHNISCHE UNIVERSITÄT DARMSTADT

AG GEOMETRIE UND APPROXIMATION

SCHLOSSGARTENSTRASSE 7

D-64289 DARMSTADT

GERMANY

alex@mathematik.tu-darmstadt.de 


\title{
PACIFIC JOURNAL OF MATHEMATICS
}

\author{
msp.org/pjm
}

Founded in 1951 by E. F. Beckenbach (1906-1982) and F. Wolf (1904-1989)

\section{EDITORS}

Don Blasius (Managing Editor)

Department of Mathematics

University of California

Los Angeles, CA 90095-1555

blasius@math.ucla.edu

\author{
Paul Balmer \\ Department of Mathematics \\ University of California \\ Los Angeles, CA 90095-1555 \\ balmer@math.ucla.edu \\ Robert Finn \\ Department of Mathematics \\ Stanford University \\ Stanford, CA 94305-2125 \\ finn@math.stanford.edu \\ Sorin Popa \\ Department of Mathematics \\ University of California \\ Los Angeles, CA 90095-1555 \\ popa@math.ucla.edu
}

\author{
Vyjayanthi Chari \\ Department of Mathematics \\ University of California \\ Riverside, CA 92521-0135 \\ chari@math.ucr.edu \\ Kefeng Liu \\ Department of Mathematics \\ University of California \\ Los Angeles, CA 90095-1555 \\ liu@math.ucla.edu \\ Jie Qing \\ Department of Mathematics \\ University of California \\ Santa Cruz, CA 95064 \\ qing@ cats.ucsc.edu
}

\section{PRODUCTION}

Silvio Levy, Scientific Editor, production@msp.org

\section{SUPPORTING INSTITUTIONS}

ACADEMIA SINICA, TAIPEI

CALIFORNIA INST. OF TECHNOLOGY

INST. DE MATEMÁTICA PURA E APLICADA

KEIO UNIVERSITY

MATH. SCIENCES RESEARCH INSTITUTE

NEW MEXICO STATE UNIV.

OREGON STATE UNIV.

\author{
STANFORD UNIVERSITY \\ UNIV. OF BRITISH COLUMBIA \\ UNIV. OF CALIFORNIA, BERKELEY \\ UNIV. OF CALIFORNIA, DAVIS \\ UNIV. OF CALIFORNIA, LOS ANGELES \\ UNIV. OF CALIFORNIA, RIVERSIDE \\ UNIV. OF CALIFORNIA, SAN DIEGO \\ UNIV. OF CALIF., SANTA BARBARA
}

\author{
Daryl Cooper \\ Department of Mathematics \\ University of California \\ Santa Barbara, CA 93106-3080 \\ cooper@math.ucsb.edu \\ Jiang-Hua Lu \\ Department of Mathematics \\ The University of Hong Kong \\ Pokfulam Rd., Hong Kong \\ jhlu@maths.hku.hk \\ Paul Yang \\ Department of Mathematics \\ Princeton University \\ Princeton NJ 08544-1000 \\ yang@math.princeton.edu
}

These supporting institutions contribute to the cost of publication of this Journal, but they are not owners or publishers and have no responsibility for its contents or policies.

See inside back cover or msp.org/pjm for submission instructions.

The subscription price for 2016 is US $\$ 440 /$ year for the electronic version, and $\$ 600 /$ year for print and electronic.

Subscriptions, requests for back issues and changes of subscribers address should be sent to Pacific Journal of Mathematics, P.O. Box 4163, Berkeley, CA 94704-0163, U.S.A. The Pacific Journal of Mathematics is indexed by Mathematical Reviews, Zentralblatt MATH, PASCAL CNRS Index, Referativnyi Zhurnal, Current Mathematical Publications and Web of Knowledge (Science Citation Index).

The Pacific Journal of Mathematics (ISSN 0030-8730) at the University of California, c/o Department of Mathematics, 798 Evans Hall \#3840, Berkeley, CA 94720-3840, is published twelve times a year. Periodical rate postage paid at Berkeley, CA 94704, and additional mailing offices. POSTMASTER: send address changes to Pacific Journal of Mathematics, P.O. Box 4163, Berkeley, CA 94704-0163.

PJM peer review and production are managed by EditFLOW ${ }^{\circledR}$ from Mathematical Sciences Publishers.

\section{PUBLISHED BY}

\section{mathematical sciences publishers \\ nonprofit scientific publishing}

http://msp.org/

(C) 2016 Mathematical Sciences Publishers 


\section{PACIFIC JOURNAL OF MATHEMATICS}

Volume 282 No. $1 \quad$ May 2016

On the half-space theorem for minimal surfaces in Heisenberg space

Tristan Alex

Extending smooth cyclic group actions on the Poincaré homology sphere 9

NIMA ANVARI

A short proof of the existence of supercuspidal representations for all reductive $p$-adic groups

RAPHAËL BEUZART-PLESSIS

Quantum groups and generalized circular elements

MICHAEL BRANNAN and KAY KIRKPATRICK

Volumes of Montesinos links

KathleEn Finlinson and Jessica S. PurCell

Minimal surfaces with two ends which have the least total absolute curvature

SHOICHI FUJIMORI and TOSHIHIRO SHODA

Multiplicité du spectre de Steklov sur les surfaces et nombre chromatique

\section{PIERRE JAMMES}

$E$-polynomial of the $\mathrm{SL}(3, \mathbb{C})$-character variety of free groups

SEAN LAWTON and VicEnTE MuÑOZ

The Blum-Hanson property for $\mathscr{C}(K)$ spaces

PASCAL LEFÈVRE and ÉTIENNE MATHERON

Crossed product algebras and direct integral decomposition for Lie supergroups

KARL-HERMANN NEEB and HADI SALMASIAN Associated primes of local cohomology modules over regular rings

TONY J. PUTHENPURAKAL 